\title{
The London School of Hygiene and Tropical Medicine: a New Century of Malaria Research
}

\begin{abstract}
Eleanor M Riley
Department of Infectious and Tropical Diseases, London School of Hygiene and Tropical Medicine, Keppel St., London WC1E 7HT, UK

The global malaria situation has scarcely improved in the last 100 years, despite major advances in our knowledge of the basic biology, epidemiology and clinical basis of the disease. Effective malaria control, leading to a significant decrease in the morbidity and mortality attributable to malaria, will require a multidisciplinary approach. New tools - drugs, vaccine and insecticides - are needed but there is also much to be gained by better use of existing tools: using drugs in combination in order to slow the development of drug resistance; targeting resources to areas of greatest need; using geographic information systems to map the populations at risk and more sophisticated marketing techniques to distribute bed nets and insecticides. Sustainable malaria control may require the deployment of a highly effective vaccine, but there is much that can be done in the meantime to reduce the burden of disease.
\end{abstract}

Key words: malaria - genetics - vaccines - drug combinations - economics

The London School of Hygiene and Tropical Medicine was established in 1899, almost simultaneously with the School of Tropical Medicine in Liverpool and with the Instituto Oswaldo Cruz, during the renaissance of research on infectious and tropical diseases that followed the pioneering work of Koch, Pasteur, Manson, Ross, Cruz, and Chagas, among others. Work on malaria has been central to the London School since its inception. Patrick Manson was the first Professor of Tropical Medicine; he initiated and supported the work of Ronald Ross in India that led to the discovery of mosquito transmission of malaria and he was followed by distinguished malariologists such as Garnham, Macdonald and Bruce-Chwatt who established long-lasting collaborative research and control programmes in many malaria-endemic countries. The commitment to malaria research continues to this day, with six professorial staff, 40 other academic staff and 30 $\mathrm{PhD}$ students working on malaria, in 30 different endemic countries. The main strength of the School lies in the interdisciplinary nature of our work, with expertise from molecular genetics to health policy and economics of malaria control.

Fax: +44-20-7637.4314. E-mail: e.riley@1shtm.ac.uk Received 7 August 2000

Accepted 4 September 2000
Malaria remains as big a threat to human health, welfare and economic development as ever. Despite decades of research, and major steps forward in treatment and prevention, the death toll remains above a million per year with more than 500 million clinical cases annually. The way forward must be a multidisciplinary approach to malaria control, comprising development and deployment of new anti-malarial drugs and drug combinations, prevention of human-vector contact by use of insecticide-treated materials, improved diagnosis and case management, monitoring of malaria outbreaks and epidemic prediction, development of a sustainable infrastructure for policy making and implementation at the local level and importantly - health education. The development of a malaria vaccine must be seen as a vital contribution to this overall strategy but not as a panacea.

THE LONDON SCHOOL OF HYGIENE AND TROPICAL MEDICINE (LSHTM)

LSHTM is a financially independent postgraduate medical school within The University of London. The School has three complementary roles: (i) to train future academics, researchers, policy-makers and practitioners in all aspects of public health and tropical medicine; (ii) to conduct high quality research; and (iii) to provide consultancy services. Our aim, through teaching, research and consultancy, is to contribute to the development of new tools for improving health, to disseminate this information and to influence policy in order to improve health, worldwide. 
Teaching is offered at several levels, all postgraduate. Taught courses include masters degrees (MSc) - full or part-time at LSHTM or by distance learning - and short courses (typically one week to three months duration) including for example short courses in epidemiology and statistics, tropical nursing, the Diploma in Tropical Medicine and Hygiene and refresher courses in infectious diseases. Research degrees include PhD, DrPH, MPhil and MRes (Master of Science by Research). In the 1999-2000 academic year there were 512 students from 82 different countries studying on 20 different masters courses at LSHTM and 257 students from 70 countries registered for research degrees.

The costs of studying full time in London are rising all the time and are beyond the reach of many students from overseas. This is borne out for example by the number of Brazilian students currently registered at LSHTM, which has fallen from 28 in 1992 to 7 in the academic year 1999-2000. In order to fulfill our mission of making our courses available to everyone who could benefit from them, we have developed two new initiatives to reach out to overseas students. The first of these is the Distance Learning Programme, which currently offers diploma and masters level training in three areas Health Systems Management, Infectious Diseases and Epidemiology; there are currently over 800 students from 44 countries registered on these courses. The second initiative is the 'split' $\mathrm{PhD}$ where overseas students can spend time in their home institution pursuing their research project; out of their 3year study time, students are required to spend only nine months in London. More details of the LSHTM teaching programme can be found at www.lshtm.ac.uk/prospectus.

Our research falls into two main categories - clinical/laboratory research and community based research. In the last UK research assessment exercise, which grades all research in UK universities, the School's research was awarded maximum scores in both of these areas. This rates our research as "internationally excellent" and places the School in the top 5 of the 40 UK medical schools. Our current re- search income is approximately $£ 17 \mathrm{~m}$ per year. The areas of research covered within the School's three departments are shown in Table I. In order to capitalise on our multidisciplinary skills, crossdepartmental research centres have been established, which cut across traditional subject areas and disciplines, to bring together a critical mass of people to work on complex problems. Examples of current research centres are shown in the Table II.

\section{MALARIA RESEARCH IN THE 21st CENTURY}

Malaria remains, along with tuberculosis and human immunodeficiency virus/acquired immunodeficiency syndrome, one of the three most important infectious diseases worldwide. The number of deaths is extremely difficult to estimate as many malaria deaths take place in the community without the patient ever coming into contact with the formal health care system, but recent estimates indicate approximately one million deaths in Africa each year, excluding deaths associated with malaria in pregnancy (Snow et al. 1999). Despite heroic efforts to control, eliminate and eradicate malaria in the middle years of the 20th century and despite some substantial advances in the last few years in developing new drugs (White et al. 1999) and new means to interrupt human-vector contact (Lengeler et al. 1998), the number of people infected with, and dying from malaria has scarcely changed. This is due in large part to the failure of these new tools to be applied in the areas where they can be of most benefit; getting research into practice has been an under-rated and under-resourced aspect of the research agenda. Nevertheless, it has become clear that only a sustained, multi-pronged approach to malaria control will have any significant impact on the disease. This realization is implicit in the approach of the 21 st century programme to reduce the burden of disease caused by malaria, the Roll Back Malaria programme of the World Health Organization, which endeavours to use whatever combination of available tools is best suited to controlling malaria in a specific location rather than relying on one or two tools and applying them everywhere (Nabarro \& Tayler 1998, Nabarro 1999).

TABLE I

Research Units at the London School of Hygiene and Tropical Medicine

\begin{tabular}{lll}
\hline $\begin{array}{l}\text { Department of Epidemiology and } \\
\text { Population Health }\end{array}$ & $\begin{array}{l}\text { Department of Public Health } \\
\text { and Policy }\end{array}$ & $\begin{array}{l}\text { Department of Infectious and Tropical } \\
\text { Diseases }\end{array}$ \\
\hline $\begin{array}{l}\text { Cancer and Public Health } \\
\text { Centre for Population Studies }\end{array}$ & $\begin{array}{l}\text { Environmental Epidemiology } \\
\text { Health Policy }\end{array}$ & $\begin{array}{l}\text { Immunology } \\
\text { Infectious Disease Epidemiology }\end{array}$ \\
$\begin{array}{l}\text { Epidemiology } \\
\text { Maternal and Child Epidemiology } \\
\text { Medical Statistics }\end{array}$ & Health Promotion Research & $\begin{array}{l}\text { Pathogen Molecular Biology and Biochemistry } \\
\text { Disease Control and Vector Biology } \\
\text { Clinical Sciences }\end{array}$ \\
\hline
\end{tabular}


TABLE II

Cross-departmental Research Centres

European Centre on Health of Societies in Transition European Observatory on Health Care Systems Water and Environmental Health at London and Loughborough

Malaria Centre

Collaborative Centre for Economics of Infectious Disease

Centre for Ageing and Public Health

Centre on Globalisation, Environmental Change and Health

Centre on Alcohol and Health

Whilst funders and policy makers must take some of the blame for the slow uptake of new research findings, researchers themselves must share the responsibility. Research must be aimed, ultimately, at delivering tools that are appropriate and affordable. This requires extensive and frequent dialogue between basic researchers and those working at the more applied end of the spectrum to make sure that research priorities continue to meet the needs of the malaria control community. Several new initiatives - Roll Back Malaria, the Multilateral Initiative on Malaria, the Medicines for Malaria Venture, the Malaria Vaccine Initiative - have all recently taken this on board and are promoting truly integrated, multidisciplinary approaches to research (Alonso \& Dgedge 1999).

\section{MALARIA RESEARCH AT LSHTM}

LSHTM is well placed to take advantage of this multidisciplinary - and inter-disciplinary atmosphere as malaria research within the School is about as broad-based as it is possible to be. In addition to basic research programmes in population genetics, molecular biology, biochemistry, immunology and cell biology, the School also hosts two major programmes funded by the UK Department for International Development (DfID) - the Malaria Consortium which, jointly with the Liverpool School of Tropical Medicine, provides consultancy services for malaria control, and a programme of applied and operational research in malarious countries of Africa and Asia, with special emphasis on the evaluation, development and implementation of insecticide-treated mosquito nets; prediction and prevention of malaria epidemics associated with climatic variation; trials of new drugs and candidate vaccines, and the epidemiology of urban malaria. Our research income has just received a significant boost from a major investment from the UK Medical Research Council for a programme of work on the relationship between malaria transmission intensity and disease burden (in collaboration with the National Institute for Medical Research and the Kilimanjaro Christian Medical Centre in Tanzania) and a five year programme from the Bill and Melinda Gates Foundation for a multinational programme on the treatment and prevention of malaria.

In order to facilitate interactions between such a disparate group of researchers, the LSHTM Malaria Centre was established in 1998, under the leadership of Professor Brian Greenwood and myself. The Centre currently comprises approximately 50 academic staff and $30 \mathrm{PhD}$ students. The aims of the Centre are to facilitate malaria research, to develop links between scientists from different disciplines, to facilitate links between the School and colleagues in malaria endemic areas, to offer training in malaria research and control and thereby to contribute to global efforts to control malaria. Details of current areas of research within the Malaria Centre, and the principal investigators for each of these areas, are available at http:// www.lshtm.ac.uk/centres/malaria.

In order to give some idea of the breadth of malaria activities at LSHTM, I have selected six areas of research to present in more detail.

\section{POPULATION GENETICS OF THE PARASITE AND ITS VECTORS}

Malaria parasites, mosquito vectors and human hosts are all genetically diverse; interactions among these three populations are affected by the prevailing genotypes of each population. The most obvious manifestations of parasite polymorphisms are anti-malarial drug resistance and antigenic diversity. The role of allelic variation in genes encoding immunodominant antigens of the parasite is hotly debated but it seems clear that antigenic polymorphism can lead to immune evasion. The data are most convincing for evasion of cell-mediated immunity polymorphisms cluster around immunodominant $\mathrm{T}$ cell epitopes (Good et al. 1988), single amino acid substitutions in $\mathrm{T}$ cell epitopes lead to loss of $\mathrm{T}$ cell recognition (Zevering et al. 1994) or antagonism of $\mathrm{T}$ cell responses (Plebanski et al. 1999) and nonsynonymous mutations out-number synonymous mutations within $\mathrm{T}$ cell epitopes (Hughes et al. 1995). Polymorphisms that allow the parasite to evade host immune responses will tend to be maintained by frequency dependent (or balancing) selection i.e. the allele will be favoured when it is rare, because very few people will have developed a specific immune response to the protein encoded by it, and the frequency of the allele will increase. When a sufficiently large proportion of the population have been infected with this parasite genotype and become immune to it, the allele will be selected against and its frequency will fall. 
Allele frequency distributions can be used to detect balancing selection (Conway 1997). Using this approach, David Conway and colleagues have identified a polymorphic region near the 5 ' end of the merozoite surface protein 1 (MSP1) gene of Plasmodium falciparum that appears to be under balancing selection and has gone on to show that naturally acquired $\mathrm{IgG}$ antibodies to this $\mathrm{N}$ terminal region of the MSP1 protein are strongly associated with resistance to clinical malaria in a malaria exposed population (Conway et al. 2000). This has interesting implications for the development of MSP1 vaccines as it has previously been thought that protective antibodies specifically target the $\mathrm{C}$ terminus of MSP1 (Egan et al. 1995, Guevara Patino et al. 1997).

David Conway's group has also used a population genetic approach to obtain more reliable estimates of the historical spread of P. falciparum. Using polymorphisms within the mitochondrial DNA (mtDNA) sequence they have demonstrated that global populations of $P$. falciparum emerged from an ancestral population within the last 50,000 years. This population diverged within Africa, with one version of the mtDNA sequence being carried to the east to give rise to $P$. falciparum populations in Southeast Asia and another version of the sequence moving west in Africa and eventually being carried to South America (Conway et al. unpub. data). Studies of this kind have important implications for understanding the spread of other parasite traits, for example drug resistance, and for unraveling both historical and current interactions between host and parasite in genetically diverse populations.

Mosquito populations are also amenable to population mapping using genetic markers. In some areas of low, and particularly seasonal, malaria transmission, larviciding is used as a method of malaria control. During the dry season, mosquitoes survive in a relatively small number of refugia typically permanent water bodies - and are thus vulnerable to elimination by larvicidal chemical sprays. The success of this approach depends upon how quickly the area is repopulated during the following wet season by mosquitoes originating outside the control area. Cally Roper has been using microsatellite markers of Anopheles gambiae $s l$ to characterize mosquito populations in different areas of KwaZulu-Natal in South Africa. Significant differences in the allele frequencies at different microsatellite loci have been found in different parts of the region, indicating that mosquito populations are subdivided and sub-populations are isolated from each other. The implication of this finding is that migration of mosquitoes within the region is limited and dry season larviciding may therefore be an effective means of vector control.
ANTI-MALARIAL DRUGS AND MALARIA TRANSMISSION

Most anti-malarial drugs act against the asexual, intra-erythrocytic stages of the parasite, having little or no effect on sporozoites, liver stages or gametocytes. The artemisinin group of anti-malarial drugs, derived from the Chinese herbal remedy qinghao or sweet wormwood, has however been reported to have gametocytocidal effects (Kumar $\&$ Zheng 1990) and may thus, if widely used, either alone or in combination with other antimalarials, have an impact on malaria transmission (Price et al. 1996). In The Gambia, Lorenz von Seidlein, a PhD student at LSHTM, has compared the efficacy of a combination of the artemisinin derivative artesunate with sulphadoxine/pyrimethamine (SP) with SP alone and has shown that the combination is safe and highly efficacious (von Seidlein et al. 2000). A follow-up study aims to see whether mass chemotherapy at the start of the malaria season can delay or prevent the annual increase in malaria cases by removing the source of infection for newly emerged mosquitoes.

Geoff Targett, Chris Drakely and Colin Sutherland have directly addressed the question of whether certain combinations of anti-malarial drugs can interfere with transmission of malaria to mosquitoes. They have compared the effect of four different drug combinations on the prevalence of gametocytes in the four weeks following treatment and have shown that treatment with SP is associated with up to three-fold higher prevalence of gametocytes post treatment compared to treatment with chloroquine (von Seidlein et al. 2000, Targett et al., unpub. data). This indicates that changing the first line drug from chloroquine to SP in endemic countries could be associated with an increase in the infectious reservoir and possibly higher levels of transmission. If these gametocytes arise from drug resistant clones of malaria, the spread of drug resistance may also be facilitated; this issue is currently being addressed at LSHTM. Importantly, this study showed that the combination of SP with artesunate reduced the prevalence of gametocytaemia to levels below those seen with chloroquine. However, membrane-feeding experiments showed that the few gametocytes that were present in people treated with artesunate/SP remained infective for mosquitoes. These studies suggest that artemisinin derivatives in some way reduce the levels of circulating gametocytes but are not in fact completely gametocytocidal.

Meanwhile, novel anti-malarial drugs that specifically target gametocyte development or gametocyte survival would be of enormous benefit - particularly in areas of marginal or highly seasonal malaria transmission, where their transmis- 
sion reducing potential would be most obvious. David Baker is taking a fundamental approach to this problem, using data from the $P$. falciparum genome project (http://www.sanger.ac.uk/Projects/

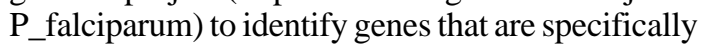
expressed during development of the sexual (gametocyte) stage of the life cycle in order to identify novel metabolic pathways that may be amenable to pharmacological manipulation (Muhia D et al. 2000). Once candidate genes have been identified, genetic transformation of malaria parasites in vitro offers a stringent method for drug target validation.

\section{MECHANISMS OF IMMUNITY TO MALARIA}

Rational design of malaria vaccines requires that we identify the targets of protective immune responses and that we determine which immune effector mechanisms actually contribute to parasite killing and resistance to clinical disease. Population genetic approaches can help to identify potential protective antigens (see above) but more direct approaches, such as immunoepidemiological studies of the association between a specific immune response to a particular antigen and susceptibility or resistance to clinical malaria, can also be used (Riley et al. 1991, Al-Yaman et al. 1994, Taylor et al. 1998). We have recently completed an immunoepidemiological study designed to test whether anti-malarial antibodies transferred from mother to child across the placenta, can in fact protect neonates and infants from malaria infection or malaria disease. Infants, under the age of 4-6 months, are well known to be relatively resistant to clinical malaria but the mechanism of resistance may be either immunological or physiological (Brabin 1990). In a prospective, longitudinal study of a birth cohort of infants in southern Ghana, we were unable to find any association between levels, antigenic specificity or antibody isotype/subclass of maternal antibody and resistance of the infants to malaria infection (Riley et al.2000). However, the overwhelming majority of malaria infections in children under six months of age were asymptomatic and of very low density (Wagner et al. 1998); infants clearly possess mechanisms for preventing parasite replication and these may well be independent of maternally acquired antibody.

An alternative explanation for the lack of clinical symptoms of malaria in infants, especially in children who developed significant levels of parasitaemia, may be that the typical febrile response to infection - which is mediated by inflammatory cytokines such as TNF $\alpha$ - is at least partially immune-mediated and dependent upon amplification by the acquired (T cell-mediated) immune response (Riley 1999). If so, then primary malaria infections in infants may be silent infections - which may remain undiagnosed and untreated - and may predispose to chronic infection and the eventual development of anaemia. Conversely, chronic lowgrade parasitaemia may be a prerequisite for development of anti-malarial immunity (premunition). Clearly, a better understanding of the natural history of malaria infections in small children may have implications for our understanding of acquired immunity but also for appropriate management of low grade infections in infants.

\section{MALARIA VACCINE TRIALS}

The development and evaluation of malaria vaccines is an excellent example of the need for multidisciplinary research. Vaccine development begins with basic immunology and molecular biology but, to move beyond the laboratory, the input of clinicians, epidemiologists and statisticians is essential and the involvement of policy makers and industry speeds the process greatly. Most malaria vaccine trials that have been conducted to date have necessarily involved collaboration between several research institutes, each with its own skills. The particular skills available at LSHTM include design and evaluation of clinical trials in general and vaccine trials in particular. Staff at LSHTM have been intimately involved in the design and monitoring of trials of the SPf66 vaccine in Tanzania and in The Gambia. More recently, Tom Doherty and Brian Greenwood have taken part in the first field trial of the RTS,S/SBAS2 vaccine developed by Smith Kline Beecham Biologicals in collaboration with the Walter Reed Army Institute of Research and based upon the circumsporozoite protein of P. falciparum (Stoute et al. 1997); a pilot study has shown that the vaccine is both safe and immunogenic (Doherty et al. 1999). The trial, funded by the European Union and co-ordinated by the Medical Research Council Laboratories in The Gambia, was a double blind, placebo-controlled trial in which 306 men (aged 18 to 45 years) were vaccinated, cleared of any existing malaria infections and monitored for reappearance of blood stage parasites during the following malaria transmission season. The results of this trial are due to be published shortly.

As immunity was believed to be at least partially cell-mediated, and as polymorphisms in $\mathrm{T}$ cell epitopes of the CS gene are known to facilitate immune evasion (as discussed above), breakthrough infections were typed at LSHTM for polymorphisms in the regions of the gene encoding immunodominant $\mathrm{T}$ cell epitopes (Th2R/Th3R). Ali Alloueche used sequence-specific oligonucleotide probes to detect 16 different alleles of the CS gene in PCR-amplified parasite DNA obtained from vaccinated and control individuals (Alloueche et al. 2000). There was no 
significant difference in allele frequencies between the two groups and no evidence that the vaccine was selecting for breakthrough infections of a different genotype. If the vaccine is ultimately shown to protect against infection and disease in children, and introduced as a routine vaccination in endemic countries, periodic monitoring will be needed to ensure that vaccine resistant parasites are not building up in the population.

\section{IMPLEMENTATION STRATEGIES FOR INSECTICIDE TREATED BED NETS}

The use of insecticide treated bed nets (ITBNs) for prevention of contact between humans and mosquitoes is one of the most successful new approaches for malaria control in Africa; almost all trials have shown significant reductions in child mortality and a recent meta-analysis confirms these findings (Lengeler 2000). Although of limited use in some parts of the world, where the predominant vector species bite outdoors and in the evening or early morning, the argument for widespread introduction of ITBNs in sub-Saharan Africa is overwhelming. The major issue now is how to achieve this. Locally produced nets can now be bought in Tanzania, for example, for as little as $\$ 4.00$ and if well cared for may last several years. Insecticide treatment of the nets can be done at the household or community level but needs to be repeated at regular intervals; the insecticide itself (a pyrethroid) is inexpensive ( $\$ 0.30$ per net per year) and can be prepackaged in single net doses (Lines \& Zaim 2000). Cost-effectiveness analysis (see below) indicates that provision of insecticide through national health programmes is a realistic expectation, although there are unresolved issues regarding monitoring of uptake and effectiveness of dipping procedures. However, the main problem at the moment is how to achieve high levels of net use.

Social scientists and entomologists at LSHTM have been working together to resolve this problem (Lines 1996). Chris Curtis and colleagues are investigating the "mass effect" of ITBNs - i.e. are high levels of ITBN coverage within a village required to significantly reduce morbidity and mortality from malaria, or will an individual sleeping under an ITBN be protected even if his neighbours are not using ITBNs? (Maxwell et al. 1999). The answer to this question is important as it will inform strategies for net distribution. If high levels of compliance are required at the individual level to afford protection at the population level, net distribution should be regarded as a public health measure (like vaccination) and should be managed as such. Alternatively, if individual nets protect individual users, net use can be viewed as a personal choice (like contraceptive use) - in which case their uptake could be promoted through health education initiatives but their provision could be managed through the commercial sector (Schellenberg et al. 1999). Jo Lines, Kara Hanson and Caroline Jones are studying the effects of these two different approaches to ITBN distribution in Tanzania and also trying to answer the vexed questions of whether social marketing is an effective means of distributing a (relatively) expensive health product (i.e. a bednet) and whether undercutting the commercial market for nets by government subsidy or non-governmental agency donation programmes will lead to loss of sustainability.

\section{MALARIA AND ECONOMICS AT LSHTM}

One of the most effective means of getting new malaria control methods adopted and put into practice is to convince funding agencies (both governments and non-governmental organisations) that the interventions are highly cost-effective. Understanding the economics of malaria and malaria control is a central part of this process. The malaria economics group at LSHTM has been leading the way in this type of analysis.

In a study that estimated the cost per disability-adjusted life years (DALYs) averted by various malaria interventions, Anne Mills, Catherine Goodman and Paul Coleman found that in very low income countries with high malaria transmission, insecticide-treated bed nets, residual spraying and chemoprophylaxis for children, all cost less than US $\$ 100$ per DALY and thus, on the basis of international guidelines (WHO 1996), can be considered extremely cost effective interventions (Goodman et al. 1999). Importantly, the study showed that it was more cost-effective to prevent malaria in pregnancy through intermittent treatment with SP than with weekly chloroquine chemoprophylaxis. When expressed as a proportion of the annual health budget of a very low income country, residual spraying turned out to be the most expensive option (accounting for 55\% of annual health expenditure for two rounds of spraying per year) whereas provision of insecticide for people to dip their own bed nets cost less than $3 \%$ of the health budget, and intermittent treatment of pregnant women with SP and a variety of measures to improve case man-agement each cost less than $1 \%$ (Goodman et al. 2000). Cost-effectiveness analysis is now being applied to the problem of changing the first-line drug for treatment of malaria cases and to the use of combinations of two or more drugs in an attempt to increase drug efficacy and slow the spread of drug resistance. Calculations of this type are essential for rational and effective use of limited health resources in low-income countries where malaria places a major burden on health facilities. 


\section{THE FUTURE}

Reducing mortality and morbidity from malaria is a major challenge for the 21 st century. New technologies are rapidly being taken up by malaria researchers and are being successfully applied to research questions - the biotechnology revolution (Gardner 1999) and geographic information systems (GIS) are two very notable examples. In the latter case, GIS mapping is revealing a hitherto unrealized complexity of malaria transmission and morbidity at the microepidemiological level (Snow et al. 1999) and its combination with meteorological data is giving us a much better picture of the time-space coordinates of malaria outbreaks and their relationship to changing climatic and environmental conditions (Bouma et al. 1997, Craig et al. 1999). This has major implications for long term forecasting of malaria epidemics and we look forward to the time when malaria control personnel in endemic regions can access the latest malaria forecasts for their area from their desk top computers via the world wide web; this should be a reality within five years and should allow malaria outbreaks to be predicted and either prevented, interrupted (by vector control or use of transmission-blocking drugs or vaccines) or managed (through provision of sufficient and appropriate drugs to hospitals and health centres).

However, as has been all too obvious in the last one hundred years, the development of new tools is not - on its own - a guarantee of improvements on the ground. Continual communication between researchers, practitioners and policy makers is essential to make sure that the right tools are applied in the right place and that their use is acceptable, affordable and sustainable. Encouraging the development of multidisciplinary research and control teams is one way to promote such communication.

\section{ACKNOWLEDGEMENTS}

To the Director, Professor José Rodrigues Coura, for inviting me to represent LSHTM at the centenary celebrations of the Instituto Oswaldo Cruz and the Mayor and City of Rio de Janeiro for their generous hospitality and also to all the members of the Instituto Oswaldo Cruz for their warm welcome, kindness and helpfulness during my stay, especially Dr Hooman Momen, Dr Eduardo Costa and Dr Yara Maria Traub Cseko. To my colleagues at LSHTM for sharing their data with me and for correcting my many misinterpretations of their work. Any remaining faults are my responsibility, not theirs.

\section{REFERENCES}

Alonso P, Dgedge M 1999. A new impetus for malaria research and control. Parasitol Today 15: 48-49.

Alloueche A, Silveira H, Conway D, Bojang K, Doherty T, Cohen J, Pinder M, Greenwood B 2000. Highthroughput sequence typing of T-cell epitope poly- morphisms in Plasmodium falciparum circumsporozoite protein. Mol Biochem Parasitol 106: 273-282.

Al-Yaman F, Genton B, Anders RF, Falk M, Triglia T, Lewis D, Hii J, Beck H-P, Alpers MP 1994. Relationship between humoral response to Plasmodium falciparum merozoite surface antigen- 2 and malaria morbidity in a highly endemic area of Papua New Guinea. Am J Trop Med Hyg 51: 593-602.

Bouma M, Poveda G, Chavasse D, Quinones M, Cox J, Patz J 1997. Predicting high risk years for malaria in Colombia using parameters of El Niño Southern Oscillation. Trop Med Int Health 2: 1122-1127.

Brabin B 1990. An analysis of malaria parasite rates in infants: forty years after Macdonald. Trop Dis Bull 87: R1-R21.

Conway D 1997. Natural selection on polymorphic malaria antigens and the search for a vaccine. Parasitol Today 13: 26-29.

Conway D, Cavanagh D, Tanabe K, Roper C, Mikes Z, Sakihama N, Bojang K, Kremsner P, Arnot D, Greenwood B, McBride J 2000. A principal target of human immunity to malaria identified by molecular population genetic and immunological analyses. Natur Med 6: 689-692.

Craig M, Snow R, le Sueur D 1999. A climate-based distribution model of malaria transmission in subSaharan Africa. Parasitol Today 15: 105-111.

Doherty J, Pinder M, Tornieporth N, Carton C, Vigneron L, Milligan P, Ballou W, Holland C, Kester K, Voss G, Momin P, Greenwood B, McAdam K, Cohen J 1999. A phase I safety and immunogenicity trial with the candidate malaria vaccine RTS, S/SBAS2 in semi-immune adults in The Gambia. Am J Trop Med Hyg 61: 865-868.

Egan AF, Chappel JA, Burghaus, Morris JS, McBride JS, Holder AA, Kaslow DC, Riley EM 1995. Serum antibodies from malaria-exposed people recognise conserved epitopes formed by the two epidermal growth factor motifs of $\mathrm{MSP}_{1}$, the carboxy-terminal fragment of the major merozoite surface protein of Plasmodium falciparum. Infect Immun 63: 456-466.

Gardner M 1999. The genome of the malaria parasite. Curr Opin Genet Dev 9: 704-708.

Good MF, Pombo D, Quakyi IA, Riley EM, Houghten RA, Menon A, Alling DW, Berzofsky JA, Miller LH 1988. Human T cell recognition of the circumsporozoite protein of Plasmodium falciparum. Immunodominant $\mathrm{T}$ cell domains map to the polymorphic regions of the molecule. Proc Natl Acad Sci USA 85: 1199-1203.

Goodman C, Coleman P, Mills A 1999. Costeffectiveness of malaria control in sub-Saharan Africa. Lancet 354: 385-387.

Goodman C, Coleman P, Mills A 2000. Economic analysis of malaria control in sub-Saharan Africa. Strategic Research Series, Global Forum for Health Research, Geneva, in press.

Guevara Patino JA, Holder AA, McBride JS, Blackman MJ 1997. Antibodies that inhibit malaria merozoite surface protein-1 processing and erythrocyte invasion are blocked by naturally acquired human anti- 
bodies. J Exp Med 186: 1689-1699.

Hughes M, Hughes A 1995. Natural selection on Plasmodium surface proteins. Mol Biochem Parasitol 71: 99-113.

Kumar N, Zheng H 1990. Stage-specific gametocytocidal effect in vitro of the antimalaria drug qinghaosu on Plasmodium falciparum. Parasitol Res 76: 214-218.

Lengeler C 2000. Insecticide-treated bednets and curtains for preventing malaria. Cochrane Database of Systematic Reviews 2: CD000363.

Lengeler C, Armstrong-Schellenberg J, D’Alessandro U, Binka F, Cattani J 1998. Relative versus absolute risk of dying reduction after using insecticide-treated nets for malaria control in Africa. Trop Med Int Health 3: 286-290.

Lines J 1996. Mosquito nets and insecticides for net treatment: a discussion of existing and potential distribution systems in Africa. Trop Med Int Health 1: 616-632.

Lines J, Zaim M 2000. Insecticide products: treatment of mosquito nets at home. Parasitol Today 16: 91-92.

Maxwell C, Myamba J, Njunwa K, Greenwood B, Curtis C 1999. Comparison of bednets impregnated with different pyrethroids for their impact on mosquitoes and on re-infection with malaria after clearance of pre-existing infections with chlorproguanil-dapsone. Trans $R$ Soc Trop Med Hyg 93: 4-11.

Muhia D, Carucci D, Witney A, Warhurst D, Schaap P, Meima M, Li J-L, Taylor M, Kelly J, Baker D 2000. Guanylyl cyclase activity associated with putative bifunctional integral membrane proteins in Plasmodium falciparum. J Biol Chem 275: 1-10.

Nabarro D 1999. Roll back malaria. Parassitologia 41: 501-504.

Nabarro D, Tayler E 1998. The "roll back malaria" campaign. Science 280: 2067-2068.

Plebanski M, Lee E, CM H, Flanagan K, Gilbert S, Gravenor M, Hill A 1999. Altered peptide ligands narrow the repertoire of cellular immune responses by interfering with T cell priming. Nat Med 5: 565571.

Price R, Nosten F, Luxemburger C, ter Kuile F, Paiphun L, Chongsuphajaisiddhi T, White N 1996. Effects of artemisinin derivatives on malaria transmissibility. Lancet 347: 1654-1658.

Riley EM 1999. Is T cell priming required for initiation of pathology in malaria infections? Immunol Today 20: 228-233.

Riley EM, Jakobsen PH, Allen SJ, Wheeler JG, Bennett S, Greenwood BM 1991. Immune responses to soluble exoantigens of Plasmodium falciparum may contribute to both pathogenesis and protection in clinical malaria: evidence from a longitudinal, prospective study of semi-immune African children. Eur J Immunol 21: 1019-1025.

Riley E, Wagner G, Ofori M, Wheeler J, Akanmori B, Tetteh K, McGuinness D, Bennett S, Nkrumah F,
Anders R, Koram K 2000. Lack of association between maternal antibody and protection of African infants from malaria infection. Infect Immun (in press).

Schellenberg J, Abdulla S, Minja H, Nathan R, Mukasa O, Marchant T, Mponda H, Kikumbih N, Lyimo E, Manchester T, Tanner M, Lengeler C 1999. KINET: a social marketing programme of treated nets and net treatment for malaria control in Tanzania, with evaluation of child health and long-term survival. Trans $R$ Soc Trop Med Hyg 93: 225-231.

Snow R, Craig M, Deichmann U, le Sueur D 1999. A preliminary continental risk map for malaria mortality among African children. Parasitol Today 15: 99-104.

Snow RW, Craig M, Deichmann U, Marsh K 1999. Estimating mortality, morbidity and disability due to malaria among Africa's non-pregnant population. Bull WHO 77: 624-640.

Stoute JA, Slaoui M, Heppner G, Momin P, Kester KE, Desmons P, Wellde BT, Garcon N, Kryzch U, Marchand M, Ballou WR, Cohen JD 1997. A preliminary evaluation of a recombinant circumsporozoite protein vaccine against Plasmodium falciparum malaria. New Engl J Med: 86-91.

Taylor RR, Allen SJ, Greenwood BM, Riley E 1998. IgG3 antibodies to Plasmodium falciparum merozoite surface protein 2 (MSP2): increasing prevalence with age and association with clinical immunity to malaria. Am J Trop Med Hyg 58: 406-413.

von Seidlein L, Milligan P, Pinder M, Bojang K, Anyalebechi C, Gosling R, Coleman R, Ude J, Sadiq A, Duraisingh M, Warhurst D, Alloueche A, Targett G, McAdam K, Greenwood B, Walraven G, Olliaro P, Doherty T 2000. Efficacy of artesunate plus pyrimethamine-sulphadoxine for uncomplicated malaria in Gambian children: a double-blind, randomised, controlled trial. Lancet 29: 352-357.

Wagner G, McGuinness D, Koram K, Bennett S, Nkrumah FK, Riley E 1998. High incidence of asymptomatic malaria infections in a birth cohort of children under 1 year of age in Ghana, detected by multicopy gene polymerase chain reaction. Am J Trop Med Hyg 59: 115-123.

White N, Nosten F, Looareesuwan S, Watkins W, Marsh K, Snow R, Kokwaro G, Ouma J, Hien T, Molyneux M, Taylor T, Newbold C, Ruebush T, Danis M, Greenwood B, Anderson R, Olliaro P 1999. Averting a malaria disaster. Lancet 353: 1965-1967.

WHO - World Health Organization 1996. Investing in health research and development: report of the Ad Hoc Committee on Health Research relating to future intervention options. Anon. TDR/Gen/96.1.

Zevering Y, Khamboonruang C, Good M 1994. Natural amino acid polymorphisms of the circumsporozoite protein of Plasmodium falciparum abrogate specific human CD4+ T cell responsiveness. Eur J Immunol 24: $1418-1425$. 